\title{
Erratum to: A novel GSH responsive poly(alpha-lipoic acid) nanocarrier bonding with the honokiol-DMXAA conjugate for combination therapy (vol 63, pg 307, 2020)
}

Zhilin Liu ${ }^{1,2}$, Zhaohui Tang ${ }^{1,2^{*}}$, Dawei Zhang ${ }^{1}$, Jiatan $\mathrm{Wu}^{3}$, Xinghui $\mathrm{Si}^{1,2}, \mathrm{Na} \mathrm{Shen}^{1}$ and Xuesi Chen ${ }^{1,2 *}$

In the version of the article originally published in the volume 63, issue 2, 2020 of Sci China Mater (2020, 63 (2): 307 315, https://doi.org/10.1007/s40843-019-1183-0), the affiliations of two of the authors (Zhaohui Tang and Xuesi Chen) were incompletely labeled. The corrected version of the authors' affiliations is as below:

Zhilin Liu ${ }^{1,2}$, Zhaohui Tang ${ }^{1,2^{*}}$, Dawei Zhang ${ }^{1}$, Jiatan $\mathrm{Wu}^{3}$, Xinghui $\mathrm{Si}^{1,2}, \mathrm{Na}$ Shen ${ }^{1}$ and Xuesi Chen ${ }^{1,2^{*}}$

\footnotetext{
${ }^{1}$ Key Laboratory of Polymer Ecomaterials, Changchun Institute of Applied Chemistry, Chinese Academy of Sciences, Changchun 130022, China

${ }^{2}$ University of Science and Technology of China, Hefei 230026, China

${ }^{3}$ Department of Chemistry, Northeast Normal University, Changchun 130021, China

* Corresponding authors (emails: ztang@ciac.ac.cn (Tang Z); xschen@ciac.ac.cn (Chen X))
} 